\title{
Some Key Developments in Computational Electromagnetics and Their Attribution
}

\author{
C. W. Trowbridge ${ }^{1}$ and J. K. Sykulski ${ }^{2}$ \\ ${ }^{1}$ Vector Fields Ltd., Kidlington, Oxford OX5 1JE, U.K. \\ ${ }^{2}$ School of ECS, University of Southampton, Southampton SO17 1BJ, U.K.
}

\begin{abstract}
Key developments in computational electromagnetics are proposed. Historical highlights are summarized concentrating on the two main approaches of differential and integral methods. This is seen as timely as a retrospective analysis is needed to minimize duplication and to help settle questions of attribution.
\end{abstract}

Index Terms-Attribution, computer aided analysis, electromagnetics, historical review, numerical analysis.

\section{INTRODUCTION}

$\mathbf{S}$ ETTING the record straight should be perceived as a subjective exercise and therefore highly personal. The authors' excuse is that they have a very long view of the work of the community and one of them has been actively involved since the earliest days of the digital computer evolution, 1957. This survey concentrates in the main on methods for field computation and ignores the very important developments in design optimization.

\section{HISTORICAL ORIGINS}

The art of field computation gathered momentum in the latter part of the twentieth century with the ever increasing power of the digital computers; though it must be said that the developments are firmly rooted in the past, e.g., the use of pencil and paper techniques, analog devices and mechanical machines to evaluate fields mapped onto meshes. Indeed, many of the ground rules can be found in the distant past culminating in the work of Southwell using finite differences in the 1940s [1].

The research activity in CEM has evolved alongside the modern developments in the digital computing hardware, and the primary motivation-quite properly-has been to serve the requirement of engineering in the production of useful devices of benefit to society. Governments and industry have been the providers of funds for this research-a fact often overlooked by those whose motivation is to develop tools for their own sake. However, we must not deny that CEM is also a scientific discipline that is capable of sustaining itself and producing new and far reaching ideas. As an example, consider the development of the finite element method (FEM) which grew out of the structural mechanics community serving the aircraft industry [2]. Its development, though based on classical principles, was driven by the needs of the industries involved and was only much later studied by mathematicians who made the connections with mainstream theory which led to a deeper understanding [3]. Thus, we had an extremely successful empirical technique, innovated and developed by engineers,

Digital Object Identifier 10.1109/TMAG.2006.872491 exploiting analogs between real discrete elements and finite regions of the continuum.

\section{PiONEERING DEVEloPMENTS}

In the meantime electrical engineers had, in the main, followed and applied the advances in finite differences (FD), a highly developed discipline of mathematics, and were able to write elegant computer codes particularly for simple static two dimensional configurations with linear media-for example, the work of Trutt at Delaware [4], Erdelyi et al. at Colorado [5], and Viviani [6] et al. in Genoa, eddy current applications by Stoll in Southampton [7], and in three dimensions by Muller and Wolf at AEG Telefunken, Germany [8]. A major difficulty with FD, in its classical form, is that the discretization schemes (mesh, grids, etc) have a fixed topology (order and arrangement) although the actual geometric distributions of mesh lines can be irregular. A further limitation arises when it is required to introduce higher order terms for the basic Taylor's series to improve the accuracy of approximation. All of these difficulties were elegantly surmounted by the finite element approach but it must be noted that FD continued to be used particularly for high frequency time domain problems in the explicit form (FDTD) first proposed by Yee [9] (1966) and later generalized by Weiland [10] (1977).

An important milestone in the solution of electromagnetics field problems came in 1963 with the seminal work of Winslow [11] at the Lawrence Livermore Laboratory California, as Winslow's papers make clear his developments were built on even earlier work by NacNeal [12] and Leith [13], they developed a discretization scheme based on an irregular grid of plane triangles, not only by using a generalized finite difference scheme but also by introducing a variational principle which led to the same result. This latter approach can be seen as being equivalent to the FEM and is accordingly one of the earliest examples of this technique used for electromagnetics. The key idea was to construct a secondary mesh of, in the case illustrated shaded in Fig. 1(a), twelve-sided figures whose vertices are alternately the centroids of the six adjacent triangles and the midpoints of the six adjacent sides. With this mesh discretized equations were readily obtained by applying gauss' theorem (finite difference approximation) or the variational principle (finite elements). In fact the work is in the spirit of the "edge" element or "Whitney forms" approach applied several years later by 


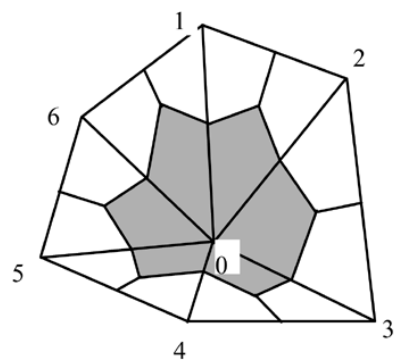

(a)

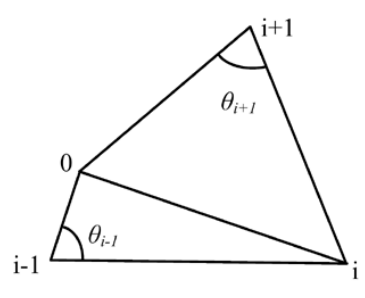

(b)
Fig. 1. Irregular triangular discretization.

Bossavit [32], but was far less general. The discretized form for the magnetostatic problem derived by Winslow using the vector potential $\mathbf{A}$ is $\sum_{1}^{6} w_{i}\left(A_{i}-A_{0}\right)=I$, where $I$ is the total current flowing through the dodecagon surrounding the point at which $A_{0}$ is defined and $w_{i}=1 / 2\left(\lambda_{i+1} \cot \left(\theta_{i+1}\right)+\lambda_{i-1} \cot \left(\theta_{i-1}\right)\right)$ and couple two adjacent triangles, see Fig. 1(b), cyclically around the pivot node of the mesh cell, the quantities $\theta_{i+1}$ and $\theta_{i-1}$ are the included angles subtended at the vertices of mesh points $i+1$ and $i-1$ respectively and $\lambda$ is the corresponding reluctivity. ${ }^{1}$ A further innovation in this work was the automatic mesh generator in which an irregular triangular mesh was constructed by solving Laplace's equation, i.e., a regular hexagonal lattice is distorted to conform to the interfaces and boundaries of the problem, thus eliminating the need for special equations at these places.

The important advantages of finite elements were now being exploited, i.e., the ease of modeling complicated boundaries and the extendibility to higher order approximations [14], and then, in 1970, came the first application of the method to rotational electrical machines by Chari and Silvester [15] (see Fig. 2). Silvester and his co-workers at McGill University developed the method in a more general way using unstructured meshes and generic higher order elements. The polynomials introduced by Silvester [16] using simplex coordinates allowed most of the mathematical formulation to be accomplished once and for all for a prototypal triangle. It is interesting to note that expressions similar to the above, involving the cotangents of the included angle at the vertices, enter the finite element matrices as weighting factors. From this time the method became widespread leading to generalized applications for time dependent and 3-D problems [17]-[20]. A parallel development to the above has been with integral methods; these integral formulations, unlike differential formulations which solve the defining partial differential equations, use the corresponding integral equation forms. These techniques became known as "the moment method" and were described theoretically by Harrington in 1968 [21]. In this context the pioneering work of Halacsy based on a point dipole approximation must be cited [22], indeed he gathered together many of the early pioneers at a series of small conferences held at Reno, Nevada (1968-1973). This approach was later generalized to include 3-D modeling and non linear materials by the Rutherford Laboratory group [23].

Yet another class of integral procedures is the so called boundary element methods [24], [25] based on applications of

\footnotetext{
${ }^{1}$ Notation as in original paper, (2) is also given in terms of edge vectors.
}

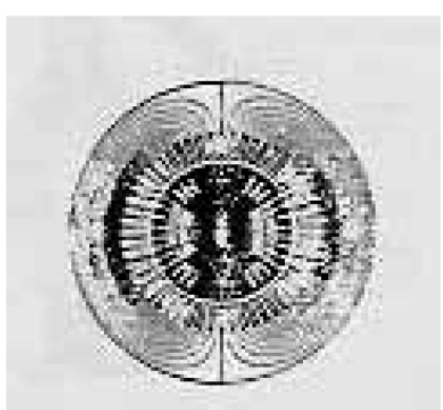

(a)

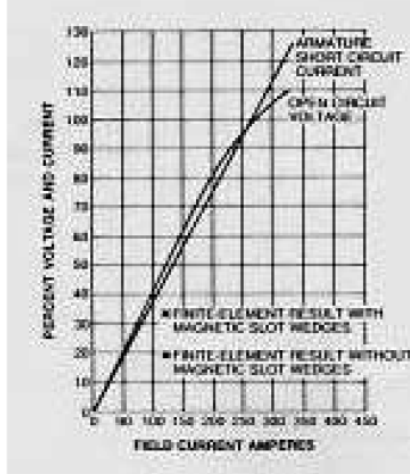

(b)
Fig. 2. Flux plots and test comparisions of a turbo generator [15].

Green's integral theorems. Whilst these methods are often difficult to apply they can produce accurate economic solutions and have been used extensively in both static and time dependent problems.

In the 1970s the various strands that made up the CEM community came together at conferences designed to exchange ideas between researchers in academia, national laboratories, and industry. The year 1976 was especially significant as in April the first Compumag Conference was held in Oxford. In that year a remarkable meeting took place in Sta Margherita under the auspices of University of Genoa and the International Journal for Numerical Methods in Engineering. Many of the leading developers of the day were present and a special book was later published [26] which summarized the status of field computation in the late 1970 s.

\section{OUTSTANDING ACHEIVEMENTS}

There have been many achievements over the last thirty years including the following.

\section{A. ICCG Method}

Pre-conditioned conjugate gradient method known as incomplete Cholesky conjugate gradient method (ICCG) when used for solving large sparse systems of equations. In this approach the operation count goes approximately $n \log n$ and is largely independent of bandwidth. This method was first introduced by Meijerink and Van der Vorst in 1977 [27] and, though not completely general as the algorithm is limited to system matrices having "property M,"2 however for the discretized electromagnetic equations this condition is usually satisfied. In this context the method was first tried in solution of 3-D magnetostatics with remarkable success [19] and rapidly taken up by most groups.

\section{B. Delaunay Meshing}

Another breakthrough has been in the now widespread use of the "Delaunay" method of generating triangular meshes [28]. The original algorithm dates from as long go as 1934 but the idea was taken up by Cendes in 1983 [29] and successfully applied to 2-D problems with mesh adaption; the Delaunay method was

\footnotetext{
${ }^{2} \mathrm{~A}$ matrix $A=a_{i j}$ is an $M$-matrix if $a_{i j} \leqslant 0$ for $i \neq j, A$ is nonsingular and $A^{-1} \geqslant 0$.
} 
soon generalized to 3-D using tetrahedral elements with error analysis [30].

\section{Kelvin Transformation}

In many situations the far field boundary is at a large distance from the active region in practical field problems, i.e., boundaries at infinity have to be taken into account. Whilst this is a natural condition with the integral method, for the differential (FEM) case this poses a difficult problem for which many methods have been proposed. An elegant solution was reported by Freeman and Lowther in 1988 [31] and independently, but in a less developed form, by Xiuying and Guangzheng [32], using mapping techniques based on the classic Kelvin transformation in which $r r^{\prime}=a^{2}$ where $r$ and $r^{\prime}$ are the inverse points with respect to a sphere of radius $a$. An inversion in a sphere is one-to-one except that the centre of the sphere of inversion has no corresponding point. The neighborhood of the origin maps into a set of points at a large distance into an infinite domain. This transformation has been used in a number of FE systems to model the infinite domain in which the exterior space to a sphere (circle) surrounding the actual model is solved as an interior problem. The nodes of the two spaces, now bounded by circles, are connected by forcing their solution values to be identical. The method has been modified and extended to 3-D problems by Imhoff, Meunier and Sabonnadiere (1990) [33].

\section{Automatic Cutting of Multiply Connected Regions}

To ensure the uniqueness of potentials in FE calculations appropriate cuts have to be introduced to ensure single valued potentials. Developments of automatic algorithms to achieve this is essential for reliable codes and has been investigated by several researchers, e.g., Simkin (1985) [34], Kotiuga (1987) [35], Kettunen (1998) [36], and Dular (2004) [37].

\section{E. The Introduction of "Edge Elements" and Differential Forms}

Whereby physical conditions are satisfied with respect to continuity conditions, "curl" and "divergence," has added to our understanding of fundamentals. These elements were first introduced to the CEM community by Bossavit [38], [39] who has been a prime mover in theoretical developments in the CEM community, though the fundamental ideas were first introduced by mathematicians, particularly the work of Whitney in 1957 [40] after whom these elements are named. They were rediscovered by the finite element community where they were defined and applied under the category of "mixed elements" by Nedelec (1980) [41]. Subsequent work by Bossavit and Verite [38], Mur and de Hoop [42], Biro et al. [43] and Yioultsis and Tsiboukis [44] demonstrated the effectiveness of these approaches in solving CEM problems. During this period 1980-1990 serious attention was being given to the role of differential forms as an alternative to Vector Calculus in formulating field problems [45]. Differential forms have been used to express Maxwell's equations since early in the last century, but many of the advantages of forms as a tool for applied electromagnetics have only recently been realized. It is claimed that, relative to the usual vector calculus treatment, differential forms make elementary electromagnetics clearer, simpler, and more intuitive. At the same time differential forms are a powerful tool for research and open the way for the application of modern differential geometry to electromagnetics. There is no doubting the economy of the expressions compared to the customary formulation in terms of vector calculus but there is also a gain in clarity and physical understanding. For instance the association of the geometric quantities of length, area and volume to the forms are compatible with the way quantities are measured, whereas the corresponding vector quantities are point densities relying on limits. Thus the essential differences between $\mathbf{B}$ and $\mathbf{H}$ are made manifest.

\section{F. Dual Energy Methods}

The algebra of differential forms provides a natural mathematical language for electromagnetics. The complexity of Maxwell's equations is reduced and the relationships can be illustrated by simple diagrams. These diagrams highlight the importance of the constitutive equations which are seen to associate energy density with infinitesimal volumes and therefore energy with complete electromagnetic systems. This gives rise to dual energy formulations. There have been several contributions in this area and one should mention in particular works by Hammond and Penman [46], Rikabi [47], and Tonti [48]. Variational methods may be applied, based on the equilibrium energy, providing either a minimum or-through a dual-complementary principle - a maximum for the system energy. This leads to a very elegant treatment in static fields providing upper and lower bounds of system parameters such as resistance, inductance and capacitance. In time-varying fields a symmetry of electrodynamics known as duality rotation becomes useful. Dual finite-element formulations are of particular interest to our community [49] as is the analysis of error bounds [50]. A geometrical approach known as the method of tubes and slices [51] is an alternative technique for solving electromagnetic problems numerically without the need to solve a large system of simultaneous equations.

\section{G. Material Modeling}

Of major interest to CEM community is how to model efficiently and accurately magnetic hysteresis and anisotropy. Various techniques have been proposed of which the most widely used are those based on scalar or vector Preisach [52] models. The fundamental work in this area has been undertaken by Mayergoyz [53], and Della Torre [54], [55]. The original vector model introduced in [53] was subsequently generalized by defining a new type of projection for the applied field vector on each direction corresponding with one scalar model [56]. Models based on neural networks have also been introduced more recently (see for example [57]). A very comprehensive review of past and present modeling techniques may be found in [58]. But material modeling is not just about magnetic hysteresis. New types of materials have emerged in recent years and require appropriate computational models. Of the many developments two are mentioned here because of their impact. The first are soft magnetic composites made from powder [59]. Advances with bonding agents, pressing technology and heat treatment have improved the properties to such an extent 
that they are serious contenders for all electrical machine applications. The benefits are lower cost and faster production, improved thermal performance, higher frequency capability leading to either higher speeds or reduced mass, volume and material requirement. The second recent advances are high temperature superconductors, which offer tremendous potential in terms of reducing the size and increasing efficiency of devices. However, they present a significant modeling challenge because of very high nonlinearity and anisotropic properties [60].

\section{H. Computation of Electromagnetic Forces}

Most commonly used methods for force calculation are based on either the Maxwell stress tensor (MST) or the virtual work principle (VWP). MST is classically derived by starting from the Lorentz force expression, whereas VWP is based on the mechanical concepts of forces being related to the change in stored energy. A comprehensive analysis of force formulations, and their implications, is given in [61]. The major benefit of MST is that it requires only a single solution of the problem but there are severe implementation problems inherent in applications to real numerical modeling systems. The VWP, on the other hand, computes the force on a body by a virtual displacement and the change in the co-energy of the system. However, the gradient of the co-energy function is normally not easily available and at least two field solutions are required. Variations of the two approaches have been considered by many authors in an attempt to improve the accuracy and reduce the computational effort. Of major note are the works of Coulomb [62], McFee [63], Kameari [64], and Hameyer et al. [65]. A recent attempt is also worth mentioning of a force computation algorithm based on continuum design sensitivity analysis [66]. The approach can generate global forces as well as force distributions over the surface of a body, including a case of zero air gap. Moreover, the force expression clearly indicates the contributions to the global force from each source of magnetic field. The implementation is simple and is independent of the numerical analysis approach taken.

\section{Moving Systems}

Several attempts have been made to solve the moving mesh problem that arises when applying FE to dynamical systems. Special airgap elements have been proposed by Razek et al. [67] involving the coupling of analytic solutions for the air-gap with a standard FE solution. A more general technique has been developed by Rodger et al. (1990) [68] which use independent FE meshes that are free to rotate and translate and which are coupled using Lagrange multipliers. The problem of overlapping meshes have been discussed by Tsukerman [69] and moving band techniques by Demenko [70].

\section{J. Fast Multipole Methods}

In solving integral equations which have an intrinsic computational cost of $O\left(n^{3}\right)$ or, if iterative methods are used, of $O\left(n^{2}\right)$ can be made far more efficient by using the "fast multipole method" introduced by Rokhlin et al. [71]. This approach tends to $O(n \log n)$ and exploits the fact that only matrix-vector products are needed in an iterative solution. Many workers see this development as pivotal in exploiting the advantages of integral equations at a reasonable computational cost. In the fast multipole method the system matrix is exactly computed in the "near field" only. The far field is obtained from the truncated local expansion in spherical polar coordinates. The terms in the multipole expansion are computed using an adaptive hierarchical scheme introduced by Greengard and Roklin [71]. The method has been applied to several problems in electromagnetics by Ruckert et al. [72] including the 3-D magnetostatic volume integral equation. This equation was first solved for 3-D problems in 1972 [23] and thus realizing the potential of the integral approach that motivated the work at Rutherford over 30 years ago.

\section{K. High Frequency Methods}

It has to be said that the FEM has not had the same impact for high frequency problems, at least in its classical form using differential operators. Indeed a wide range of methods have been used to match the broad range of applications encountered. For instance the method of moments (MOM) [21], based on the integral operator, is well suited to the open boundary problems involved in scattering and antenna applications. The advantage of this method is that meshing is not required in the free space regions. However, dense sets of equations are involved and, unlike the FEM, the solution time is of order $n^{3}$, so we must expect to see the FMM method discussed above having a strong impact in this area. One advantage is that the inefficient procedure of diminishing the mesh size to achieve a desired accuracy as the wavelength decreases is largely avoided.

\section{Transmission Line Matrix Method}

Other methods used for high frequency problems include the circuit analogy method, transmission line matrix (TLM), first introduced by Johns [73] which in effect is a numerical implementation of the classical Huygens's principle for the modeling of wave propagation and is ideally suited to time discretization but not so effective in modeling geometry. Electrical engineers have an intuitive understanding of how electrical circuits work and are often more comfortable with circuit models than with the more abstract mathematics normally used to study electromagnetic fields. TLM is now a well established technique for modeling EM problems [74], although current implementations are restricted to structured Cartesian meshes. Unstructured meshes offer better versatility for mapping complex geometries and hence current effort is directed to develop TLM algorithms capable of working with these types of unstructured triangular meshes [75]. Unstructured meshes are particularly beneficial where a range of feature sizes need to be accurately modeled and easily lend them to nonuniform meshing, employing the necessary computational resources around small features whilst minimizing computational effort in the empty spaces.

\section{Finite Difference Time Domain Methods (FDTD)}

One development that has emerged in the last two decades for high frequency applications has been the success of finitedifference time-domain method [9] first proposed by Yee in 
1966 which, though theoretically equivalent to the TLM method [76], is both easier to implement and easier to adapt to complex boundaries. The classic result achieved by setting $c \Delta t / \Delta x=1$ in the time discretization scheme ensures that the second order FD equations are exactly satisfied. Furthermore stability is guaranteed by choosing $\Delta t \leqslant \Delta x / c$. The method uses central differences to evaluate the derivatives in the two first "order" Maxwell "curl" equations by using two interlacing meshes and obtain a second order accurate FDTD scheme. The two meshes are offset by half the grid size for the $\mathbf{E}$ and $\mathbf{H}$ fields respectively. However, the method is severely tested when nonorthogonal meshes are needed to model complex geometries and indeed to deal with open boundaries. Much of the vast literature in FDTD is concerned with extending the method to deal with these situations [77], e.g., work of the team at the Warsaw University of Technology in which "conforming" meshing technique is used which allows the modeling of curved surfaces without the "staircase" problem usually encountered in orthogonal grids [78].

\section{N. Finite Integration Method}

This approach was introduced by Weiland in 1977 [10] and developed significantly in the following years [79]. This formulation also utilizes two interlacing grids, one each for the first and second Maxwell equations respectively. However, the usual field components are replaced by their exact integrals along lines or over areas. The interesting feature of the method being that the set of matrix equations obtained is a consistent representation of the original field equations when mapping from real space to the discretized space and thus can be applied to problems across the frequency spectrum. The discretized approximation arises only through the constitutive relations rather than the difference expressions for the derivatives. Various methods for time discretization have been used including the "leap frog" scheme which in the case of a regular grid conforms to the Yee method. There are clear theoretical links between finite integration and mixed finite element methods [80].

\section{CONCLUSION}

There is a significant body of publications in the area of CEM appearing each year in a variety of journals and presented at many conferences. It is important that effort is not duplicated and fundamental work is referenced. This contribution is a personal view of the authors but hopefully goes some small way toward achieving this goal. We suggest that the International Compumag Society takes up the challenge of formulating an objective portfolio of the key developments in the subject to facilitate further research.

\section{REFERENCES}

[1] R. Southwell, Relaxation Methods in Theoretical Physics: OUP, 1946.

[2] M. J. Turner et al., "Stiffness and deflection analysis of complex structures," J. Aero Sci., vol. 23, p. 805, 1956.

[3] J. T. Oden, "A general theory of finite elements," Int. J. Numer. Meth. Eng., vol. 1, 1969.

[4] F. C. Trutt, "Analysis of homopolar inductor alternators," Ph.D. thesis, Univ. Delaware, 1962.

[5] E. A. Erdelyi and S. V. Ahmed, "Non-linear theory of synchronous machines on load," IEEE Trans. Power App. Syst., vol. PAS-85, p. 792, 1966.
[6] G. Molinari et al., "Finite difference method with irregular grid and transformed discretization metric," presented at the IEEE PES Winter Mtg., 1978.

[7] R. L. Stoll, The Analysis of Eddy Currents. Oxford, U.K.: Clarendon, 1974.

[8] W. Muller and W. Wolff, "General numerical solution of the magnetostatic equations," AEG Telfunken, Tech. Rep. 49(3), 1976.

[9] K. S. Yee, "Numerical solution of initial boundary value problems involving Maxwell's equations in isotropic media," IEEE Trans. Antennas Propag., vol. AP-14, pp. 302-307, 1966.

[10] T. Weiland, "A discretization method for the solution of Maxwell's equations for six component fields," Electron. Commun. (AEU), vol. 31, p. 116, 1977.

[11] A. M. Winslow, "Numerical calculation of static magnetic fields in an irregular triangle mesh," J. Comput. Phys. 1, p. 149, 1966.

[12] R. H. MacNeal, "An asymetrical finite difference network," $Q$. Appl. Math., vol. 11, p. 295, 1953.

[13] C. E. Leith and L. R. L. Livermore, California, CA, 1958. Unpublished.

[14] O. C. Zienkiewicz and R. Taylor, The Finite Element Method, 4th ed. New York: McGraw-Hill, 1991.

[15] M. V. K. Chari and P. P. Silvester, "Finite element analysis of magnetically saturated dc machines," IEEE Trans. Power App. Syst., vol PAS-89, no. 7, pp. 1642-1651, 1970. vol. PAS-90, No 2, pp. 454-464 1971.

[16] P. P. Silvester, "High-order polynomial triangular finite elements for potential problems," Int. J. Eng. Sci., vol. 7, pp. 849-861, 1969.

[17] C. J. Carpenter, "Comparison of alternative formulations of 3-D magnetic field and eddy current problems at power frequencies," Proc. IEE, vol. 124, no. 11, 1977.

[18] J. L. Coulomb, A. Konrad, J. C. Sabonnadiere, and P. Silvester, "Finite element analysis of steady state effect in a slot-embedded conductor," IEEE WPM A76-189-1, 1976.

[19] J. Simkin and C. W. Trowbridge, "On the use of the total scalar potential in the numerical solution of field problems in electromagnetics," Int. J. Numer. Meth. Eng., vol. 14, p. 432, 1978.

[20] T. Nakata and N. Takahashi, "Direct finite element analysis of flux and current distributions under specified conditions," IEEE Trans. Magn., vol. MAG-18, no. 2, pp. 325-330, Mar. 1982.

[21] R. F. Harrington, Field Computation by Moment Methods. New York: Macmillan, 1968.

[22] A. A. Halacsy, Proc. 2nd Reno Conf. Analysis of Magnetic Fields, Reno, NV, 1969 , p. 56

[23] M. J. Newman, C. W. Trowbridge, and L. R. Turner, "GFUN: An interactive program as an aid to magnet design," in Proc. 4th Int. Conf. Magnet Technology, Brookhaven, NY, 1972.

[24] M. A. Jaswon, "Integral equation methods in potential theory," Proc. $R$. Soc. A, p. 23, 1963.

[25] J. Simkin and C. W. Trowbridge, "Magnetostatic fields computed using an integral equation derived from Green's theorem," presented at the Compumag Conf. Computation of Magnetic Fields, 1976.

[26] M. V. K. Chari and P. P. Silvester, Eds., Finite Elements in Electrical and Magnetic Field Problems. New York: Wiley, 1980.

[27] J. A. Meijerink and V. der Vorst, "An iterative solution method for systems of which the coefficient matrix is a symmetric M matrix," Maths. Comp., vol. 31, p. 148, 1977.

[28] B. Delaunay, "Sur la sphere vide," Izves. Akad. Nauk. USSR, Math. and Nat Sci. Div., no. 6, p. 793, 1934.

[29] Z. Cendes et al., "Magnetic field computation using Delaunay triangulation and complementary finite element methods," IEEE Trans. Magn., vol. MAG-19, no. 6, pp. 2551-2554, Nov. 1983.

[30] L. Janucke and A. Kost, "Error estimation and adaptive mesh generation in the 2D and 3D finite element method," IEEE Trans. Magn., vol MAG-32, no. 3, pp. 1334-1337, May 1996.

[31] E. M. Freeman and D. A. Lowther, "A novel mapping technique for open boundary finite element solutions to Poisson's equation," IEEE Trans. Magn., vol. 24, no. 6, pp. 2934-2936, Nov. 1988.

[32] Q. Xiuying and N. Guangzheng, "Electromagnetic field analysis in boundless space by finite element method," in Compumag Graz Conf. Rec., IGTE, 1987.

[33] J. Imhoff, G. Meunier, and J. C. Sabonnadiere, "Finite element modeling of open boundary problems," IEEE Trans. Magn., vol. 26, no. 2, pp. 588-591, Mar. 1990.

[34] C. Harrold and J. Simkin, "Cutting multiply connected domains," IEEE Trans. Magn., vol. MAG-21, no. 6, pp. 2495-2498, Nov. 1985.

[35] R. Kotiuga, "On making cuts for magnetic scalar potentials in multiply connected regions," J. Appl. Phys., vol. 61, no. 8, pp. 3916-18, 1987. 
[36] L. Kettunen, K. Forsman, and A. Bossavit, "Formulation of the eddy current problem in multiply connected regions in terms of h," Int. J. Numer. Meth. Eng., vol. 41, 1998.

[37] P. Dular, "Curl-conform source fields in finite element formulations," COMPEL, vol. 24, no. 2, 2005.

[38] A. Bossavit and J. C. Verite, "A mixed FEM-BIEM method to solve 3-D eddy current problem," IEEE Trans. Magn., vol. MAG-18, no. 2, pp. 431-435, Mar. 1982.

[39] A. Bossavit, "Whitney forms: A class of finite elements for three-dimensional computations in electromagnetism," IEE Proc. A, vol. 135, pp. 493-500, 1988.

[40] H. Whitney, Geometric Integration Theory. Princeton, NJ: Princeton Univ. Press, 1957.

[41] J. C. Nedelec, "Mixed finite elements in R3," in Numerische Mathematic: Springer-Verlag, 1980, vol. 35, pp. 316-341.

[42] G. Mur and A. de Hoop, "A finite element method for computing three-dimensional electromagnetic fields in inhomogeneous media," IEEE Trans. Magn., vol. MAG-21, no. 6, pp. 2188-2191, Nov. 1985.

[43] O. Biro, K. Preis, and K. Richter, "On the use of the magnetic vector potential in the nodal and edge finite element analysis of 3D magnetostatic problems," IEEE Trans. Magn., vol. 32, no. 3, pp. 651-654, May 1996.

[44] T. Yioultsis and T. Tsiboukis, "Multiparametric vector finite elements: A systematic approach to the construction of 3-D, higher order, tangential vector shape functions," IEEE Trans. Magn., vol. 32, no. 3, pp. 1389-1392, May 1996.

[45] D. Baldomir, "Differential forms and electromagnetism in 3-dimensional Euclidian space $\mathrm{R}^{3}$," IEE Proc. A, vol. 133, p. 139, 1986.

[46] P. Hammond and J. Penman, "Calculation of inductance and capacitance by means of dual energy principles," Proc. IEE, vol. 123, no. 6 , pp. 554-559, Jun. 1976.

[47] J. Rikabi, C. Bryant, and E. Freeman, "Error based derivation of complementary formulations for the eddy-current," Proc IEE, pt. A, vol. 135, pp. 208-216, 1988.

[48] E. Tonti, "Finite formulation of electromagnetic field," ICS Newsletter, vol. 8, no. 1, pp. 5-11, 2001.

[49] Z. Ren, "Application of differential forms in the finite element formulation of electromagnetic problems," ICS Newsletter, vol. 7, no. 3, pp. $6-11,2000$.

[50] R. Albanese, R. Fresa, and G. Rubinacci, "Error bounds in computational electromagnetics," ICS Newsletter, vol. 5, no. 3, pp. 4-6, 1998.

[51] P. Hammond and J. K. Sykulski, Engineering Electromagnetism: Physical Processes and Computation. Oxford, U.K.: Oxford Univ. Press, 1994.

[52] F. Preisach, Z. Phys., vol. 94, p. 277, 1935.

[53] I. M. Mayergoyz, "Mathematical models of hysteresis," IEEE Trans. Magn., vol. MAG-22, no. 5, pp. 603-608, Sep. 1986.

[54] E. Della Torre, INTERMAG Conf., Phoenix, AZ, 1985.

[55] A. Reimers et al., "Implementation of the preisach DOK magnetic hysteresis model in a FE package," IEEE Trans. Magn., vol. 37, no. 5, p. 3362, Sep. 2001.

[56] A. Adly and I. Mayergoyz, "A new vector Preisach type model of hysteresis," J. Appl. Phys., vol. 73, pp. 5824-5826, 1993.

[57] M. Kuczmann and A. Ivanyi, "A new neural-network-based scalar hysteresis model," IEEE Trans. Magn., vol. 38, no. 2, pp. 857-860, Mar. 2002.

[58] L. Dupre and J. Malkebeek, "Electromagnetic hysteresis modeling: From material science to finite element analysis of devices," ICS Newsletter, vol. 10, no. 3, pp. 4-14, 2003.

[59] A. G. Jack, B. C. Mecrow, P. G. Dickinson, and D. Stephenson, "Permanent-magnet machines with powdered iron cores and prepressed windings," IEEE Trans. Ind. Appl., vol. 36, no. 4, pp. 1077-1084, Jul.-Aug. 2000.

[60] J. K. Sykulski, K. F. Goddard, and R. L. Stoll, "A method of estimating the total AC loss in a high-temperature superconducting transformer winding," IEEE Trans. Magn., vol. 36, no. 4, pp. 1183-1187, Jul. 2000.
[61] C. J. Carpenter, "Surface-integral methods of calculating forces on magnetized iron parts," IEE Monograph no. 342, pp. 19-28, 1959.

[62] J. L. Coulomb and G. Meunier, "Finite element implementation of virtual work principle for magnetic force and torque computation," IEEE Trans. Magn., vol. MAG-20, no. 5, pp. 1894-1896, Sep. 1985.

[63] S. McFee, J. Webb, and D. A. Lowther, "A tunable volume integration formulation for force calculation in finite-element based computational magnetostatics," IEEE Trans. Magn., vol. 24, no. 1, pp. 439-442, Jan. 1988.

[64] A. Kameari, "Local force calculation in 3D FEM with edge elements," in Nonlinear Phenomena in Electromagnetic Fields. Amsterdam, The Netherlands: Elsevier, 1992, pp. 449-452.

[65] F. Henrotte, H. V. Sande, G. Deliege, and K. Hameyer, "Electromagnetic force density in a ferromagnetic material," IEEE Trans. Magn., vol. 40, no. 2, pp. 553-556, Mar. 2004.

[66] D. H. Kim David, A. Lowther, and J. K. Sykulski, "Efficient force calculations based on continuum sensitivity analysis," IEEE Trans. Magn., vol. 41, no. 5, pp. 1404-1407, Sep. 2005.

[67] A. Razek, J. Coulomb, M. Feliachi, and J. C. Sabonnadiere, "Conception of an air-gap element for the dynamic analysis of the electromagnetic field in electric machines," IEEE Trans. Magn., vol. MAG-18, no. 2, pp. 655-659, Mar. 1982.

[68] D. Rodger, H. Lai, and P. Leonard, "Coupled elements for problems involving motion," IEEE Trans. Magn., vol. 26, no. 2, pp. 548-550, Mar. 1990.

[69] I. A. Tsukerman, "Overlapping finite elements for problems with movement," IEEE Trans. Magn., vol. 28, no. 5, pp. 2247-2249, Sep. 1992.

[70] A. Demenko, "Movement simulation in finite element analysis of electric machine dynamics," IEEE Trans. Magn., vol. 32, no. 3, pp. 1553-1556, May 1996.

[71] L. Greengard and V. Rokhlin, "A new version of the fast multipole method for the laplace equation in three dimensions," Acta Numerica, pp. 229-269, 1997.

[72] A. Buchau, W. Rieger, and W. Rucker, "Efficient integral equation method for the solution of 3-D magnetosatic problems," IEEE Trans. Magn., vol. 41, no. 5, pp. 1408-1411, Sep. 2005.

[73] P. B. Johns, "Application of the transmission-line matrix method to homogeneous waveguides of arbitrary cross section," in Proc. Inst. Electr. Eng., vol. 119, Mar. 1974, pp. 209-215.

[74] C. Christopoulos, The Transmission-Line Modeling Method: TLM: IEEE Press and Oxford Univ. Press, 1995.

[75] P. Sewell, J. G. Wykes, T. M. Benson, D. W. P. Thomas, A. Vukovic, and C. Christopoulos, "Transmission line modeling using unstructured meshes," in IEE Proc. SMT, vol. 151, 2004, pp. 445-448.

[76] M. Celuch-Marcysiak and W. K. Gwarek, "Generalized TLM algorithms with controlled stability margin and their equivalence with finite-difference formulations for modified grids," IEEE Trans. Microw. Theory Tech., vol. 43, no. 9, pp. 2081-2090, Sep. 1995.

[77] A. Taflove, Advances in Computational Electrodynamics. Boston, MA: Artech House, 1998.

[78] M. Celuch-Marcysiak and W. K. Gwarek, "Higher-order modeling of media interfaces for enhanced FDTD analysis of microwave circuits," in Proc. 24th Eur. Microwave Conf., Cannes, 1994, pp. 1530-1535.

[79] T. Weiland, "Time domain electromagnetic field computation with finite difference methods," Int. J. Numer. Model., vol. 9, pp. 295-319, 1996.

[80] A. Bossavit and L. Kettunen, "Yee-like schemes on a tetrahedral mesh, with diagonal lumping," Int. J. Numer. Model., vol. 12, pp. 129-142, 1999.

Manuscript received June 20, 2005 (e-mail: bill@trowbridge.org.uk). 